\title{
kyste de Gorlin : à propos d'un cas
}

RÉSUMÉ Le kyste odontogénique épithélial calcifié (K.O.C.) est une lésion bénigne classée actuellement parmi les tumeurs épithéliales avec induction du mésenchyme.

Cliniquement, le K.O.C. est en général asymptomatique, souvent c'est la tuméfaction à croissance lente qui est remarquée, découverte à l'occasion de signes inflammatoires généralement modérés. Ce kyste peut être aussi

Lamia OUALHA

AHU au serivce de Médecine et Chirurgie Buccales,

Clinique Dentaire de Monastir, rue Avicenne, 5000 Monastir, Tunisie.

\section{Hager HENTATI}

AHU au serivce de Médecine et Chirurgie Buccales,

Clinique Dentaire de Monastir, Tunisie.

\section{Souha BEN YOUSSEF}

Professeur au service de Médecine Dentaire, CHU Farhat Hached Sousse,

Tunisie.

\section{Faten BEN AMOR}

Professeur en Anatomie à la Faculté de Médecine Dentaire de Monastir, Monastir, Tunisie.

\section{Jamil SELMI}

Professeur, Chef du Service de Médecine et Chirurgie Buccales,

Clinique Dentaire de Monastir, Tunisie.

\section{Yves COMMISSIONAT}

Professeur au Collège de Médecine des Hôpitaux de Paris,

Membre de l'Académie Nationale

de Chirurgie Dentaire,

10, avenue Percier,

75008 Paris. découvert suite à un examen radiologique de routine ou chez l'enfant, en présence de troubles d'éruption.

Radiologiquement, le K.O.C. se traduit par un foyer ostéolytique radioclair de contour plus ou moins précis. Monogéodique ou polygéodique, l'image est souvent parsemée de fines ponctuations radio-opaques, périphériques, de taille et nombre variables et fréquemment en rapport avec une dent incluse, mais ces dernières peuvent faire défaut compliquant ainsi la pose d'un diagnostic précis.

Le traitement de ce kyste consiste en l'exérèse complète avec curetage de la cavité résiduelle.

Le diagnostic définitif est histologique.

MOTS CLÉS
tumeur odontogénique bénigne
cellule fantôme
kyste calcifié

Oualha L, Hentati H, Ben Youssef S, Ben Amor F, Selmi J, Commissionat Y. Kyste de Gorlin : à propos d'un cas Actualités Odonto-Stomatologiques 2007:240:347-357 


\section{introduction}

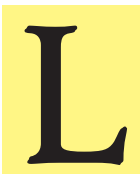

e kyste de Gorlin ou kyste odontogène calcifié (KOC) a été définitivement décrit en 1962 par Gorlin, et al. et en 1963, par Gold[1].

Il s'agit d'une lésion rare, bénigne, d'évolution lente, non douloureuse à cause de l'absence de réaction périostée.

Ce kyste est une lésion bénigne classée actuellement parmi les tumeurs épithéliales avec induction du mésenchyme.

Il est probablement issu de l'épithélium odontogénique situé dans la muqueuse ou l'os alvéolaire.

L'auteur rapporte une localisation rare de kyste odontogène calcifié, pouvant rendre le diagnostic clinique et même radiologique difficile et la liste de diagnostics différentiels plus longue.

\section{cas clinique}

Il s'agit d'un patient âgé de 42 ans, adressé au Service de Médecine et Chirurgie Buccales par son médecin dentiste pour une tuméfaction labiale supérieure persistante, évoluant depuis deux mois.

Le patient n'a pas pu confirmer la vitalité dentaire de la 11 et de la 21 avant le traitement endodontique entrepris malgré tout par son médecin dentiste.

Par ailleurs, le patient a bénéficié d'une antibiothérapie.

L'examen locorégional est normal, sans hypoesthésie cutanée, ni adénopathies.

À l'examen exobuccal on note une légère tuméfaction labiale supérieure (fig. 1).

L'examen endobuccal montre une tuméfaction vestibulaire antérieure de $2 \mathrm{~cm}$ de grand axe (fig. 2), non douloureuse à la palpation, de consistance fluctuante et recouverte par une muqueuse saine.
Le test de vitalité est négatif sur la 11 et la 22 ; positif sur toutes les autres dents maxillaires. Aucune mobilité dentaire n'a été notée.

Le panoramique montre :

1 - une image ostéolytique antérosupérieure en forme de cœur, de $2 \mathrm{~cm}$ de grand axe en rapport avec la 11 , la 21 et la 22 ;

2 - une obturation canalaire de mauvaise qualité au niveau de la 21 ;

3 - une résorption de l'apex de la 11 (fig. 3a) ;

4 - des opacités à l'intérieur de cette image :

5 -l'image ostéolytique englobe les péri-apex de la 11 , la 21 et la 22 (fig. 3b) ;

$\mathrm{Au} v \mathrm{vu}$ de cet examen clinique et radiologique, plusieurs diagnostics peuvent être évoqués devant le fait que :

- les dents étaient déjà traitées endodontiquement lors de notre consul- 
tation et la vitalité pulpaire (paramètre d'orientation diagnostique) ne peut plus être prise en considération ;

- la région anatomique où évolue la lésion donne toujours sur les clichés radiologiques une image «en cœur de carte à jouer» non spécifique, due à la présence de
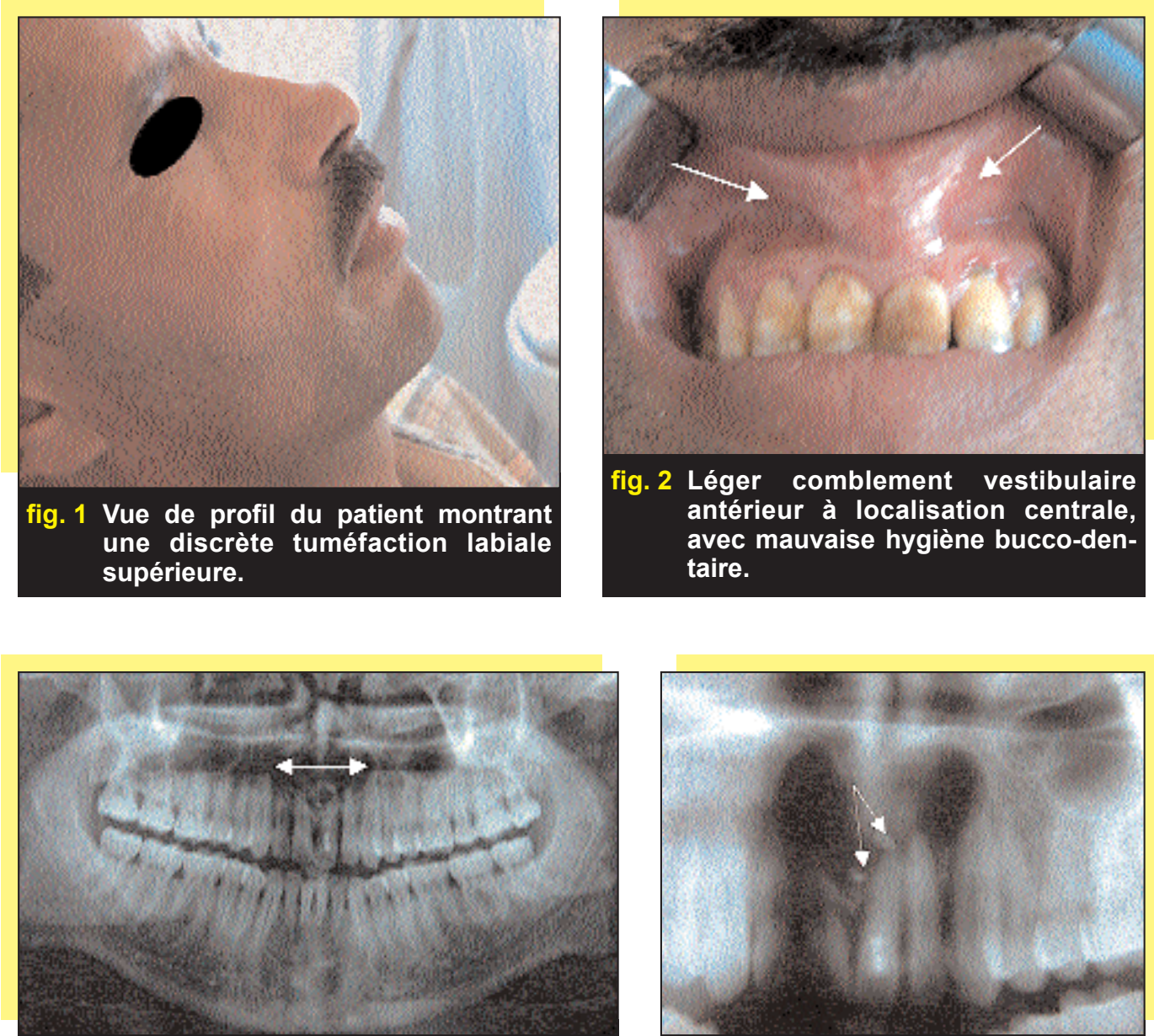

fig. 3 a Image radioclaire antéro-supérieure en regard de la 11, la 21 et la 22 avec des opacités à l'intérieur de cette image et un traitement endodontique insuffisant sur la 21. l'épine nasale antérieure et aux racines des deux incisives centrales ;

- le panoramique ne permet pas de définir la tonalité des opacités retrouvées à l'intérieur de la radioclarté : s'agit-il d'un dépassement du produit d'obturation ou bien de calcifications? 


\section{LE KYSTE FISSURAIRE}

(NASO-PALATIN)

Ce diagnostic peut être retenu si on considère que les dents étaient vivantes avant leur trépanation et que les opacités proviennent du produit d'obturation.

En plus, l'image lytique engendrée, en «cœur de carte à jouer», est typique de ce type de kyste.

Cependant, ce kyste évolue le plus souvent du côté palatin.

\section{TUMEUR ADÉNOMATOÏDE}

Cette tumeur peut être évoquée devant les cacifications radio-opaques.

En effet, l'aspect radiologique habituel est une image radioclaire, uniloculaire, bien limitée, pouvant contenir des microcalcifications radio-opaques caractéristiques, siégeant électivement dans la partie antérieure du maxillaire en regard des canines et souvent en rapport avec une dent incluse.

Cependant, ce diagnostic suppose que le médecin traitant a effectué un traitement endodontique non justifié sur des incisives centrales conservant leur vitalité pulpaire.

\section{KYSTE DE GORLIN}

Il peut être évoqué si l'on considère que les opacités sont de nature dentinocémentaire et amélaire et présentes en périphérie de la lésion.

Cependant, ce kyste est beaucoup plus fréquent en situation postérieure par rapport à la canine au niveau maxillaire.

\section{TUMEUR ÉPITHÉLIALE ODONTOGÉNIQUE CALCIFIÉE : LA TUMEUR DE PINDBORG[2]}

Au sein de l'image radiologique les opacités, lorsqu'elles existent, sont centrales, nombreuses, de nature osseuse, rondes ou allongées.

En plus, cette tumeur est généralement en rapport avec une dent incluse le plus souvent entourée par les opacités.

Son siège est surtout prémolo-molaire mandibulaire.

\section{KYSTE PRIMORDIAL SUR DENT SURNUMÉRAIRE}

Ce diagnostic suppose qu'il existe une dent surnuméraire, mais ce type kystique est plus fréquent au niveau de la région prémolo-molaire mandibulaire.

\section{FIBRO-ODONTOME AMÉLOBLASTIQUE}

Si les calcifications sont de nature dentaire et proches de celles de l'émail avec une zone ostéolytique radioclaire étendue, le diagnostic de fibro-odontome améloblastique peut être évoqué.

\section{LE FIBRO-DENTINOME AMÉLOBLASTIQUE}

C'est la tonalité des calcifications qui diffère par rapport au fibro-odontome améloblastique. Ces calcifications sont de tonalité moindre que celle de l'émail. Cependant, l'étude exacte des tonalités ne peut être faite sur un simple cliché panoramique.

\section{LE KÉRATOKYSTE[3]}

En absence de calcifications, ce diagnostic peut être évoqué. 


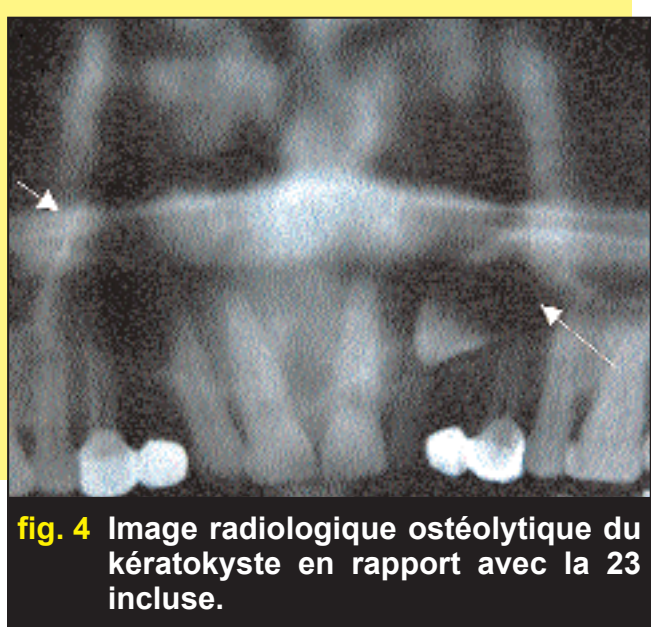

$\mathrm{Au}$ niveau maxillaire, le siège du kératokyste est le plus souvent dans la région prémolaire.

Les signes cliniques sont en rapport avec l'expansion du kyste et les déplacements dentaires qu'il provoque se confondant ainsi avec le KOC[4].

L'image radiologique du kératokyste est celle d'une géode mono- ou polyfocale, homogène, ronde ou ovale, aux contours réguliers et nets, bien tracés, voire épaissis[5] (fig. 4).

Certains kératokystes enveloppent une dent adjacente non évoluée (fig. 5) et développent la rhizalyse des autres dents situées à son contact.

\section{L'AMÉLOBLASTOME}

Ce diagnostic est aussi évoqué en absence de calcifications.

L'améloblastome peut siéger sur l'ensemble de la mandibule, mais plus préférentiellement à l'angle mandibulaire, son siège préférentiel au maxillaire étant la tubérosité.

Il entraîne souvent une mobilité dentaire et des résorptions radiculaires.

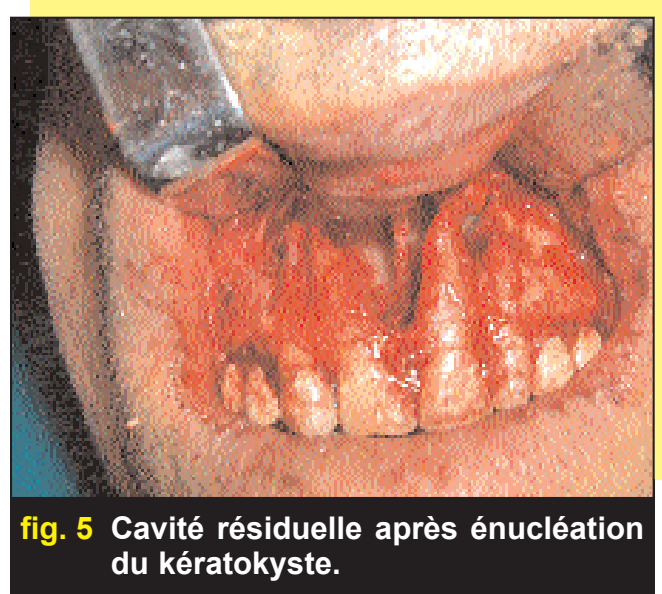

L'image radiologique est non spécifique, allant de la simple image monogéodique à celle de bulles de savon, aux contours réguliers, de taille variable.

En évoluant, ces bulles disparaissent par amincissement de leur cloison lors de l'augmentation du volume de la tumeur.

L'attitude thérapeutique a consisté à refaire le traitement endodontique de la 21 , effectuer le traitement endodontique de la 11 et enfin réaliser l'énucléation kystique sous anesthésie locale (fig. 6 à 11).

L'examen anatomo-pathologique (fig. 12 et 13) a montré que la paroi du kyste est tapissée par un épithélium multistratifié, d'aspect malpighien. Focalement, cet épithélium comporte des amas de cellules éosinophiles momifiées et parfois calcifiées. Ces cellules sont, par places, visibles dans le conjonctif sousjacent. Celui-ci est fibreux, siège d'un infiltrat inflammatoire plasmocytaire et d'amas de macrophages dont le cytoplasme est granulaire verdâtre, éléments en faveur du kyste odontogénique calcifié. 

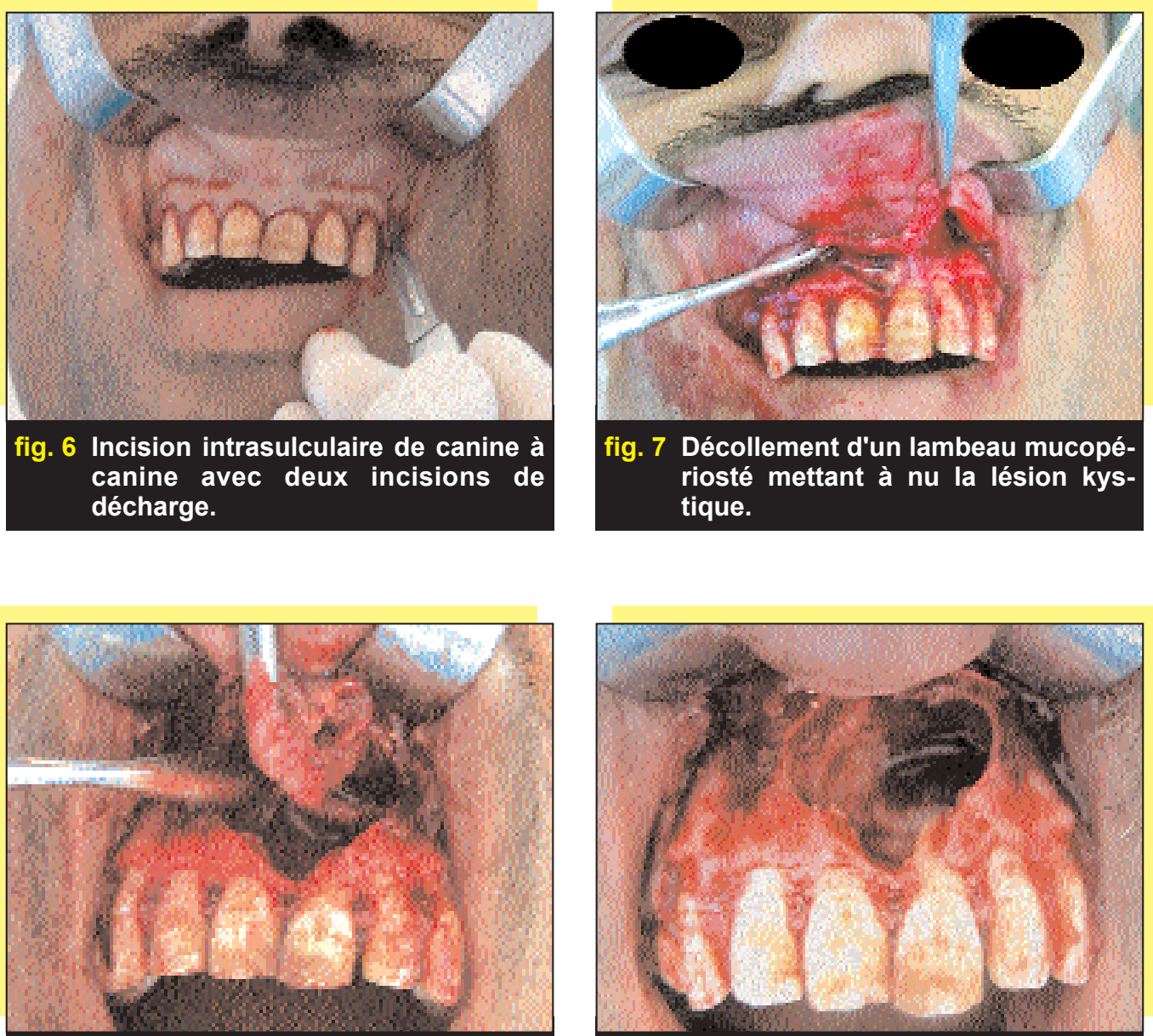

fig. 8 Énucléation de la paroi kystique.

fig. 9 Cavité résiduelle après curetage.
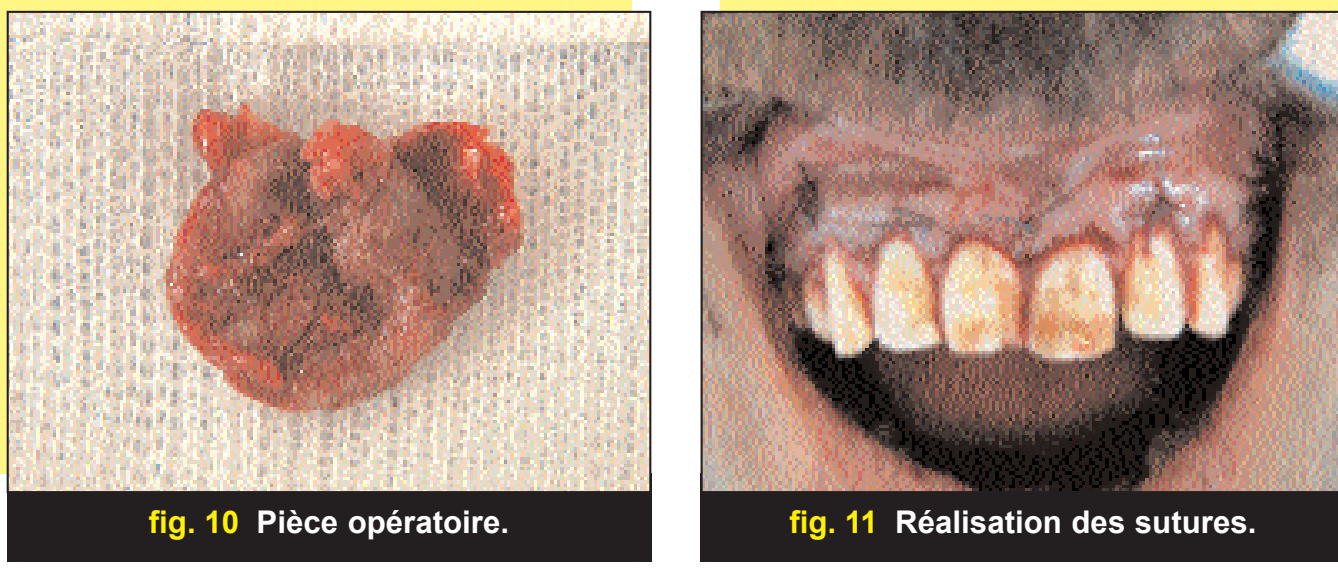

fig. 11 Réalisation des sutures. 

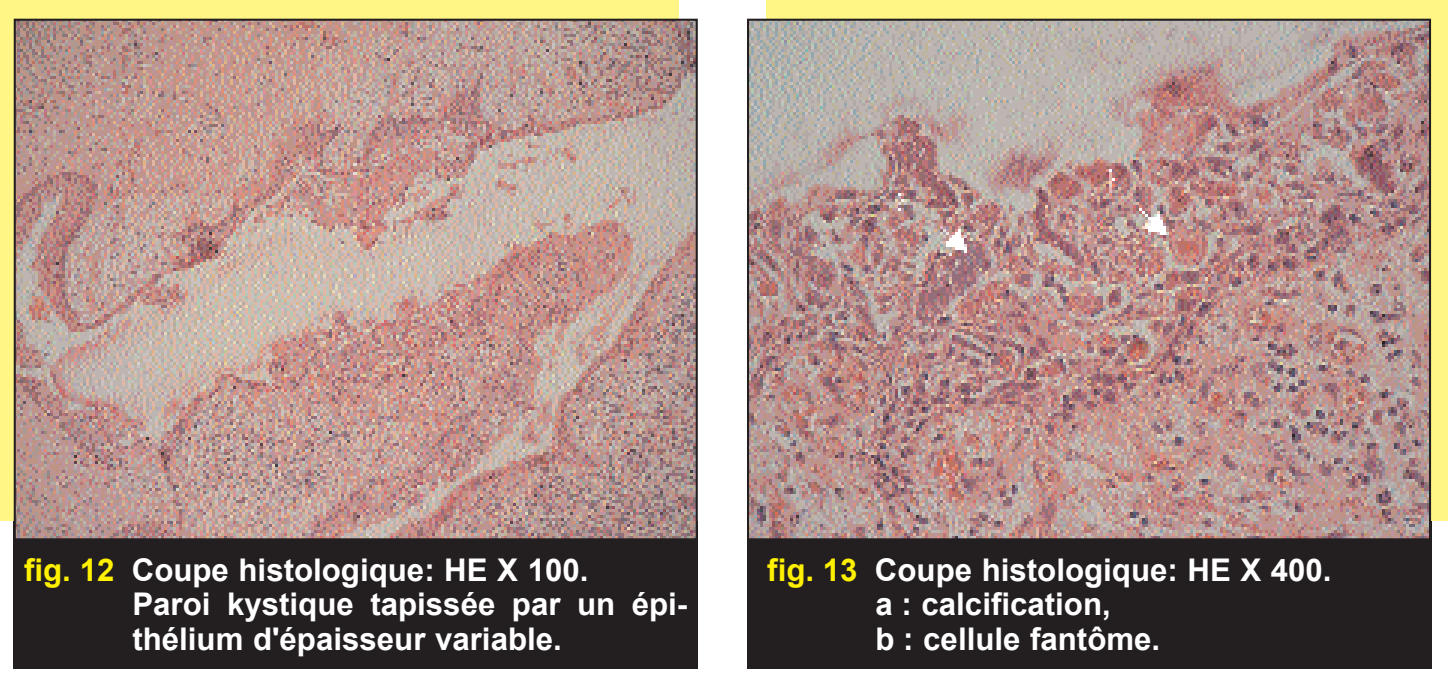

\section{discussion}

Précédemment confondu avec un améloblastome ou un odontome atypique, ce kyste est identifié par Gorlin[6].

Le kyste odontogénique calcifié est une lésion bénigne rare qui affecte les maxillaires (moins de 0,1\% des kystes des maxillaires). Il est probablement issu de l'épithélium odontogénique situé dans la muqueuse ou l'os alvéolaire.

La symptomatologie est fruste, voire inexistante, sans spécificité (traduction courante des tumeurs odontogènes et non odontogènes bénignes).

Dans notre cas, la symptomatologie s'est résumée en une tuméfaction vestibulaire antérieure.

Ce kyste survient à n'importe quelle époque de la vie, avec une légère prédilection pour les deuxième et troisième décennies.

Freedman, et al. (1975) notent une distribution différente selon l'âge : il serait plus fréquent au maxillaire jusqu'à la quatrième décade et plus fréquent à la mandibule après 41 ans. Ces résultats concordent avec notre cas.

Il affecte à proportions égales l'homme et la femme[7].

Dans $80 \%$ des cas, le kyste est intraosseux, presque toujours en zone dentée, exceptionnellement en zone édentée[1, 6].

D'autres variétés extra-osseuses, dites périphériques, sont de fréquence moindre $(20 \%)$ et décrites au niveau des parties molles gingivales recouvrant les aires d'éruption dentaires[8].

Ces dernières surviendraient chez des sujets plus âgés, adoptant ainsi certaines similitudes avec l'améloblastome périphérique ou le kyste gingival de l'adulte.

Un cas extra-osseux a été observé au sein de la glande parotide et un cas isolé au sein de la muqueuse alvéolaire mandibulaire[4, 9]. 
La localisation est, semble-t-il, autant maxillaire que mandibulaire.

La localisation maxillaire préférentielle est postérieure à la canine, contrairement au cas présenté.

La tuméfaction est non gênante pour le patient, découverte par hasard, à l'occasion de signes inflammatoires, bien souvent modérés qui marquent une poussée évolutive révélatrice[10]. Cependant, dans ce cas clinique, il n'y avait pas de signes inflammatoires mais juste une tuméfaction. La douleur est rare, elle était absente chez notre patient.

La découverte peut aussi être fortuite, au cours d'un examen dentaire de routine ou lors d'un retard d'éruption et il n'est pas rare de trouver plus d'une dent incluse en relation étroite avec le kyste.

La déformation intra-buccale est recouverte d'une muqueuse saine, parfois érythémateuse. À la palpation, les sensations sont différentes selon son stade d'évolution :

- soit fluctuante, dépressible et qui peut glisser à la mobilisation sur le plan profond sans y adhérer, d'aspect purement kystique ;

- soit ferme et solide, bien adhérante, d'aspect tumoral ;

- soit aussi de forme intermédiaire dite mixte, de transition entre la forme kystique et la forme néoplasique.

L'exploration se fait habituellement par les incidences classiques et la TDM est réservée au bilan d'extension des lésions volumineuses.

Radiologiquement, le kyste apparaît le plus souvent comme une lacune uni- loculaire bien limitée, des masses calcifiées de tailles variables sont dispersées dans la radioclarté (calcifications de petite taille dans $10 \%$ des cas et de grande taille dans $20 \%$ des cas)[1].

Dans plus de $25 \%$ des cas, le kyste est en relation avec une dent incluse. Des résorptions des racines des dents voisines attribuables à la pression $d u$ kyste ont été observées. Alors que, dans le cas présenté, les dents adjacentes ne présentaient pas de résorptions.

Le traitement recommandé est l'énucléation chirurgicale. Les récidives sont très rares.

Sa dégénérescence maligne a été exceptionnellement observée, marquée par des infiltrations et des métastases.

Histologiquement, le kyste possède un épithélium composé d'une assise basale cuboïdale ou cylindrique surmontée par deux ou trois assises de cellules d'aspect étoilé.

Dans cette couche de cellules étoilées, se trouvent des cellules dites fantômes (ghost cells), gonflées, éosinophiles, qui ont perdu leur noyau et qui contiennent beaucoup de tonofibrilles[9].

Concernant les cellules épithéliales, Shear (1983), puis Lucas (1984)[11] ont montré que le revêtement épithélial est composé d'un épithélium pavimenteux non kératinisant, linéaire ou plissé.

Il est constitué d'une assise basale cuboïdale ou cylindrique, à noyaux hyperchromatiques, surmontée par une couche superficielle d'épaisseur variable, composée de cellules basophiles aux longs prolongements cytoplasmiques, étoilées rappelant celles de l'organe de 
l'émail des dents en formation ou les améloblastomes.

Par zones, l'épithélium peut être très aminci ou, au contraire, fortement hyperplasié. Freedman, et al. (1975), puis Praetoruis, et al. (1981) ont expliqué que, dans ce dernier cas, l'aspect caractéristique du KOC ne subsiste que par zones. Les cellules épithéliales et les cellules étoilées ont un marquage net avec les anticorps anti-cytokératines. Le stroma fibreux comportant de l'ostéodentine peu différenciée d'origine mésodermique peut être observé (dentine dysplasique, d'aspect ostéoïde atypique, sans structure tubulaire normale), soit directement au contact des cellules basales, soit plus profondément sous forme de globules.

Cette ostéodentine peut être le résultat d'un effet inducteur ou simplement d'une métaplasie au sein du tissu conjonctif.

D'autres auteurs nient l'origine mésodermique éventuelle de cette dentine dysplasique, suggérant, sur base d'études ultrastructurales, qu'elle représente un type dur de kératine, similaire à celui trouvé dans les ongles.

Parfois aussi, de la dentine tubulaire et de l'émail se développent formant des structures rappelant les dents[11, 12].

Les cellules fantômes sont caractéristiques mais non pathognomoniques $d u$ kyste odontogénique calcifié[11] ; elles sont en effet observées aussi, mais plus rarement, dans les fibro-odontomes améloblastiques, dans les odontomes complexes et composés, dans les améloblastomes, dans les carcinomes et même dans les cranio-pharyngiomes ou les épithéliomas de Malherbe.

Ces cellules dites fantômes (ghost cells), éléments pâles, éosinophiles, ayant perdu leur noyau contiennent beaucoup de tonofibrilles, de 60 à $240 \mathrm{~mm}$ de diamètre, arrangés dans diverses directions[13].

Elles sont présentes dans la couche de cellules étoilées d'une qualité variable.

Pour expliquer la présence des calcifications, Fejerskow et Krogh ont bien décrit l'originalité histologique de cette tumeur avec des cellules épithéliales de type malpighien différentes et dont l'aspect ultrastructural, ainsi que les activités enzymatiques comparables à celles des cellules du stratum intermédium, leur font évoquer une origine possible de la tumeur à partir de ces cellules.

Ces cellules à potentialité odontogène ne pourraient pas produire une matrice de l'émail, mais une substance amyloïde, et les calcifications seraient alors un essai de minéralisation de cette matrice anormale.

Ces calcifications, au début, centrales, s'étendent et fusionnent pour former parfois une vaste plage. Il est admis que ceux-ci résultent de la calcification de la substance amyloïde, mais ils pourraient, pour une part, provenir de collagène calcifié ou bien de la dégénération des mitochondries ou des tonofibrilles[14].

La substance amyloïde induirait la production par les cellules du stroma d'une matrice collagène, qui se calcifierait et ressemblerait au cément. 


\section{conclusion}

Cliniquement, le KOC est le plus souvent asymptomatique, seule la tuméfaction à croissance lente est remarquée. Le traitement consiste en l'exérèse complète.

Une surveillance clinique et radiologique dont la périodicité dépend de la conclusion anatomopathologique et jusqu'à l'obtention d'une réossification complète est nécessaire.

L'examen histologique est indispensable.

Enfin, c'est de la confrontation radioclinique et histologique que naîtra le diagnostic.
Du point de vue évolutif, ce kyste guérit après énucléation complète ; d'exceptionnelles récidives locales et tardives ont cependant été signalées et mises sur le compte d'une exérèse incomplète.

De plus, une dégénérescence maligne de la variété tumorale a été exceptionnellement observée et décrite sous le nom de carcinome odontogénique à cellules fantômes, avec croissance importante et tendance à l'infiltration des tissus adjacents et, dans certains cas, des métastases.

\section{bibliographie}

1. Piette $\mathrm{E}$.

Pathologie dentaire non carieuse.

In : Piette E,

Reychler $\mathrm{H}$, eds.

Traité de pathologies

buccale et maxillo-faciale.

Bruxelles :

De Boeck Université, 1991:1238-1240.

2. Kaplan I, Pindborg JJ, Shear M.

Radiological and clinical features of calcifying epithelial odontogenic tumor. Dentomaxillofac Radiol 2001;30:22-28

3. Chomette G, Auriol M. Histopathologie buccale et cervico-faciale. Paris : Masson, 1985.
4. Shear M

Developmental odontogenic cysts.

J Oral Pathol Med 1994; 23:1-11.

5. Le Charpentier $Y$, Favre-Dauvergne $E$, Auriol M. Kystes des maxillaires. Editions Techniques Encycl Med Chir (Paris, France), Stomatologie-Odontologie I, 22-062-G-10, 1994.

6. Favre-Dauvergne $E_{\text {, }}$ Auriol M, Le Charpentier Y. Tumeurs odontogéniques. Editions Techniques Encycl Med Chir (Paris, France), Stomatologie-Odontologie I, 22-062-F-10, 1995, 10 p.
7. Johnson A $3^{\text {rd }}$, Fletcher $L$, Gold I, Chen C.

Calcifying odontogenic cyst: a clinicopathologic study of $\mathbf{5 7}$ cases with immunohistochemical evaluation for cytokeratin. J Oral Maxillofac Surg 1997;55:679-683.

8. Takeda Y, Suzuki A, Sekiyama S.

Peripheral calcifying epithelial odontogenic tumor.

Oral Surg Oral Med Oral Pathol 1983;56:71-75.

9. Orsini G, Fiorini M, Rubini C, Piatelli A. Peripheral calcifying odontogenic cyst. $\mathrm{J}$ Clin Periodontal 2002;29:83-86. 
10. McGowan RH,

Browne RM.

The calcifying

odontogenic cysts:

a problem of

preoperative diagnosis.

J Oral Surg 1982;20:

203-212.

11. Gordeeff $M$.

Kystes odontogènes.

J Biol Bucc 1989;17:

136-142.
12. Mesquita $R$, Lotufo $M$.

Peripheral clear cell variants of calcifying epithelial odontogenic tumours.

Oral Surg Med Oral Pathol Oral Radiol Endod 2003; 95:198-201.

13. Satomura $K$, Nakanishi $H$, Fujisawa K. Initiation of ectopic epithelial classification in a calcifying

odontogenic cyst.

J Oral Pathol Med 1999; 28:330-335.

14. Mimura M, Tanaka N, Kimijima.

An ultrastructural study calcifying odontogenic cyst, especially calcified material.

Med Electron Mirosc 2002; 35(2):109-116.

\section{SUMMARY}

\section{The calcifying odontogenic cyst: a case report}

L. OUALHA, H. HENTATI, S. BEN YOUSSEF, F. BEN AMOR, J. SELMI, Y. COMMISSIONAT

Calcified epithelial odontogenic cysts (C.O.C.) are benign lesions classified among the epithelial tumours with mesenchyme induction.

Clinically, C.O.C. are generally asymptomatic. They are often discovered following tumefaction of slow growth or inflammatory signs that are generally moderate. They are also discovered following a routine radiological examination or in children having troubles of eruption.

Radiologically, C.O.C. are shown by a radioclear osteolytic image, having more or less a precise contour.

Mono- or polygeodic, the image is frequently scattered with radio-opaque, peripheral fine points. They are of variable size and number. They are frequently related to unerupted teeth. However, sometimes, these points do not exist which complicates diagnosis.

The treatment of these cysts consists in the complete ablation with curettage of the residual cavity. The final diagnosis is histologic.

keywords: odontogenic benign tumors, ghost cell, calcifying cyst. 